\title{
Accuracy of 2-Phase Parathyroid CT for the Preoperative Localization of Parathyroid Adenomas in Primary Hyperparathyroidism
}

\author{
B. Griffith, H. Chaudhary, G. Mahmood, A.M. Carlin, E. Peterson, M. Singer, and S.C. Patel
}

\begin{abstract}
BACKGROUND AND PURPOSE: Minimally invasive parathyroidectomy requires accurate preoperative localization of suspected adenomas, and multiphase CT allows adenoma characterization while providing detailed anatomic information. The purpose of this study was to assess the feasibility of a protocol using only arterial and venous phases to localize pathologic glands in patients with primary hyperparathyroidism.
\end{abstract}

\begin{abstract}
MATERIALS AND METHODS: We identified 278 patients with primary hyperparathyroidism who had undergone 2-phase CT with surgical cure. All scans were read prospectively by board-certified neuroradiologists. A neuroradiology fellow retrospectively reviewed images and reports and classified suspected adenomas on the basis of anatomic location. Accuracy was determined by comparing imaging results with surgical findings. The ability of 2-phase CT to localize adenomas to 1 of 4 neck quadrants and lateralize them to the correct side was assessed. Accuracy of identifying multigland disease was also evaluated.
\end{abstract}

RESULTS: In patients with single-gland disease, the sensitivity and specificity of 2-phase CT to correctly localize the quadrant were $55.4 \%$ and $85.9 \%$, respectively. The sensitivity and specificity of correct lateralization were $78.8 \%$ and $67.8 \%$, respectively. The sensitivity and specificity to identify multigland disease were $22.9 \%$ and $79.5 \%$, respectively.

CONCLUSIONS: While the 2-phase CT protocol in this study demonstrates lower accuracy compared with reports of other techniques, its lower radiation compared with 3- and 4-phase techniques may make it a feasible alternative for preoperative parathyroid localization. Further prospective studies are needed to identify patients for whom this technique is most suitable.

$\mathbf{P}$ rimary hyperparathyroidism, a disorder caused by the presence of $\geq 1$ hyperfunctioning parathyroid gland, is characterized by the overproduction of parathyroid hormone and is the most common cause of hypercalcemia in nonhospitalized patients. ${ }^{1-3}$ Primary hyperparathyroidism is most commonly due to a single adenoma ( $85 \%)$ but can also be caused by multiple adenomas, 4-gland hyperplasia, and parathyroid carcinoma. ${ }^{2}$

The treatment of choice for primary hyperparathyroidism is surgical removal of the hyperfunctional tissue. ${ }^{1}$ In the past, surgical management required bilateral cervical exploration. Improvements in preoperative localization of abnormal glands, however, and the use of intraoperative parathyroid hormone assays have led to increased use of minimally invasive, or focused, parathy-

Received February 23, 2015; accepted after revision May 15.

From the Departments of Radiology (B.G., S.C.P.), Otolaryngology (H.C., M.S.), Surgery (A.M.C.), and Health Sciences (E.P.), Henry Ford Health System, Detroit, Michigan; and Department of Surgery (G.M.), University of Toledo, Toledo, Ohio.

Please address correspondence to Brent Griffith, MD, Department of Radiology, K3, Henry Ford Hospital, 2799 West Grand Blvd, Detroit, MI 48202; e-mail: brentg@rad.hfh.edu

http://dx.doi.org/10.3174/ajnr.A4473 roidectomy techniques. ${ }^{3,4}$ Compared with bilateral exploration, minimally invasive parathyroidectomy achieves the same outcomes while offering a lower risk profile, causing less pain, and providing superior cosmetic outcomes.

The success of minimally invasive parathyroidectomy depends on the accurate preoperative localization of a potentially hyperfunctioning gland. A number of imaging modalities are currently used, both alone and in combination, for preoperative localization, including radionuclide scintigraphy and radionuclide single-photon emission CT, sonography, and contrast-enhanced CT. Radionuclide scintigraphy and sonography have both demonstrated success in preoperative localization, but each has limitations as well. In particular, both modalities lack detail regarding surrounding anatomy, and sonography is highly operator-dependent. ${ }^{5-7}$

In the past decade, multiphase CT has emerged as an additional technique for adenoma localization. ${ }^{1,2,8-14}$ In addition to lesion identification, CT allows precise localization based on welldefined anatomic landmarks. Following its introduction, multiphase CT was referred to as $4 \mathrm{D}-\mathrm{CT} .{ }^{9}$ The term " $4 \mathrm{D}$ " referred to the 3 dimensions provided by the CT scan with the added dimen- 
sion of time due to the functional information offered by the multiple phases of imaging. ${ }^{9}$ Since the initial description of 4D-CT by Rodgers et $\mathrm{al}^{9}$ in 2006, many studies have attempted to define the optimal number of phases needed for parathyroid adenoma detection.

Two-phase parathyroid CT uses only 2 phases of imaging for characterization and localization purposes, which, in this study, included an arterial and a venous phase. Identification of parathyroid adenomas is based on the principle that parathyroid lesions show rapid enhancement followed by washout, which is demonstrated on the arterial and venous phases, respectively. The obvious major benefit of 2-phase CT over 3- or 4-phase CT is the decreased radiation dose. This is of particular importance given the increasing attention paid to radiation exposure from medical imaging. ${ }^{15}$ In addition, studies have suggested that multiple phases do not improve localization, with a recent study finding that the arterial phase alone was comparable with the combination of other phases in correctly localizing and lateralizing adenomas. ${ }^{16}$ Our institution began using this 2-phase parathyroid CT exclusively in 2009 for the preoperative work-up of parathyroid adenomas when cross-sectional imaging localization was requested by the referring surgeon.

The purpose of this study was to retrospectively assess the accuracy of 2-phase parathyroid CT for localizing surgically proved parathyroid adenomas in patients with primary hyperparathyroidism. We hypothesized that 2-phase CT would be a feasible technique for preoperative localization compared with techniques using 3 or 4 phases, while offering a reduction in radiation dose.

\section{MATERIALS AND METHODS}

This retrospective study was conducted in accordance with Health Insurance Portability and Accountability Act regulations. The study was approved by our institutional review board, and a waiver of informed consent was obtained.

\section{Patients}

Between May 2009 and August 2013, 444 two-phase parathyroid CT scans were obtained on 428 patients at our institution. Of these, 278 patients met the following inclusion criteria:

- Laboratory data consistent with primary hyperparathyroidism

- First-time parathyroidectomy surgery with subsequent cure (defined as normal calcium levels at 6 months or a 50\% drop in intraoperative parathyroid hormone levels and into the normal range).

One hundred sixty-six CT scans were excluded for the following reasons: Seventy patients underwent 1 or multiple 2-phase CT scans without surgery performed, 14 patients had operative or pathology reports that were indeterminate, 18 patients did not have a cure following surgery, 25 patients had inadequate preoperative documentation, 27 patients were either lost to follow-up or had no postoperative laboratory data, and 12 patients were undergoing repeat surgeries.

Of the 278 included patients, 250 had either radionuclide scintigraphy or radionuclide single-photon emission CT performed. Of those, 140 patients had a suspected adenoma identified on the scan.
The decision to obtain a CT scan at our institution is variable and is determined by the individual surgeons on a patient-bypatient basis. There is no differentiation of patients who had previously undergone successful or unsuccessful localization by other modalities, whether sestamibi or sonography.

\section{CT Technique}

All examinations were performed with a $16-$ or 64 -detector row CT scanner. Patients were positioned supine, head first on the CT gantry. Examinations included a scout scanogram and 2 phases of imaging following the intravenous administration of contrast material. One hundred milliliters of iopamidol (Isovue-350; Bracco, Princeton, New Jersey) or ioversol (Optiray-300; Mallinckrodt, St. Louis, Missouri) was administered at an injection rate of 5 $\mathrm{mL} / \mathrm{s}$ via an 18-ga catheter followed by $40 \mathrm{~mL}$ of saline at $2 \mathrm{~mL} / \mathrm{s}$.

Timing of the 2 phases of imaging (arterial and venous) was dependent on patient age. In patients older than 55 years, the first-phase scanning started following a 22 -second delay. The delay was 18 seconds in patients younger than 55 years of age. The second (venous) phase was obtained immediately following the first phase.

The multiphase CT was acquired at 2.5-mm section thickness with a $2.5-\mathrm{mm}$ interval. Automatic exposure control was used (range, 200-400 mA) at $120 \mathrm{kV}$ with a noise index of 13.81 . Craniocaudal coverage was from the base of the orbit to the aortic arch. The mean CT dose index per imaging phase was $24.49 \mathrm{mGy}$. Coronal and sagittal reformats were obtained for both phases at $2.5-\mathrm{mm}$ section thickness with a $2.5-\mathrm{mm}$ interval.

\section{Image Analysis}

All 2-phase parathyroid CT reports and images were retrospectively reviewed by a neuroradiology fellow, and suspected adenomas were classified on the basis of their anatomic location: right upper quadrant, right lower quadrant, left upper quadrant, and left lower quadrant. Localization was based on a combination of image annotations (eg, measurements, arrows) placed at the time of the initial interpretation and the anatomic description on the report. All initial image interpretations were performed prospectively by board-certified neuroradiologists. No images were reinterpreted by the fellow at review. Quadrants were based on lesion location with respect to the vertical midline (right and left) and a transverse plane through the middle of the thyroid gland (Fig 1). Potential adenomas were also assigned a lateralization (right or left) based on their position relative to the midline. Suspected adenomas crossing a quadrant line were localized to the quadrant where the greatest proportion of the gland was located. Nontraditional locations, including ectopic and intrathyroidal glands, were also identified.

\section{Surgical Localization}

The surgery and pathology records were reviewed by a third-year medical student and a fourth-year otolaryngology resident with all final determinations of gland location confirmed with an otolaryngology staff member specializing in parathyroid surgery. Because surgeries were performed by multiple surgeons, if surgical localization could not be confidently determined on the basis of operative and pathology reports, the patient was excluded from analysis. 


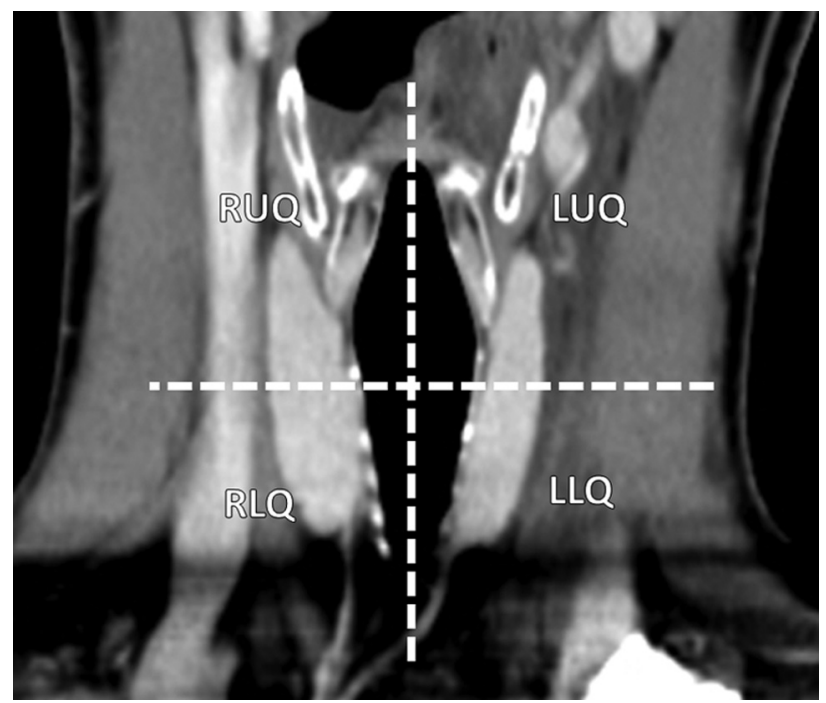

FIG 1. Coronal reformat image demonstrates the 4 quadrants used for imaging localization purposes. Quadrants were based on lesion location with respect to the vertical midline (right and left) and a transverse plane through the middle of the thyroid gland.

Gland location was classified by the same method as imaging localization. Medical records were reviewed to obtain patient demographic data, including age, sex, weight, body mass index, and preoperative and postoperative parathyroid hormone and calcium levels.

For diagnostic accuracy, surgical and pathologic findings were treated as the criterion standard.

\section{Statistical Analysis}

Suspected adenomas localized to the correct quadrant on the basis of surgical and pathologic findings were scored as true-positive. Suspected adenomas localized to the incorrect quadrant were scored as false-positive. The remaining quadrants not identified with possible adenomas were evaluated as a "no adenoma" guess - if correct on the basis of surgery, they were classified as a true-negative, and if misclassified, they were considered a false-negative. Each individual had 4 determinations, 1 for each quadrant. Screening parameters of sensitivity, specificity, positive predictive value, and negative predictive value were estimated by using a ratio estimator commonly used in sampling theory. Standard errors were also estimated. This estimation was performed for both single-gland quadrant localization and single-gland lateralization, considering only 2 areas on the 2-phase CT scan instead of 4 .

For purposes of determining the accuracy of multigland identification, true-positives were those patients with multigland disease found at surgery and suspected adenomas identified at multiple locations on CT. False-negatives were those patients found to have multigland disease at surgery but without suspected multiple glands identified on CT. True-negatives and false-positives were those patients with only a single gland found at the time of surgery and with a single suspected gland or multiple suspected glands identified on CT, respectively.

Sensitivity, specificity, positive predictive value, and negative predictive value were compared for both localization and lateralization for those patients with single-gland disease within 1 of the 4 quadrants at the time of surgery. The following variables were
Table 1: Patient demographics

\begin{tabular}{|c|c|c|}
\hline \multicolumn{2}{|l|}{ Demographics } & \\
\hline \multicolumn{2}{|l|}{ Age $(y r)(n=278)$} & $60.5 \pm 12.7$ \\
\hline \multicolumn{2}{|l|}{ Weight (kg) $(n=241)$} & $85.23 \pm 21$ \\
\hline \multicolumn{2}{|c|}{ Body mass index $(n=209)$} & $31.4 \pm 7.0$ \\
\hline \multicolumn{2}{|c|}{ Preoperative PTH level $(n=278)^{\mathrm{a}}$} & $128.5(26.7-1900)$ \\
\hline \multicolumn{2}{|c|}{ Intraoperative PTH level $(n=278)^{a}$} & $23.6(0-151.7)$ \\
\hline \multicolumn{2}{|c|}{ Preoperative calcium level ${ }^{\mathrm{a}}$} & $10.6(8.7-14.6)$ \\
\hline \multicolumn{2}{|c|}{$\begin{array}{l}\text { Multigland disease at surgery } \\
\text { (No. of patients) }\end{array}$} & 48 \\
\hline \multicolumn{2}{|c|}{$\begin{array}{l}\text { Nontraditional-location disease at } \\
\text { surgery (No. of patients) }\end{array}$} & 8 \\
\hline \multicolumn{2}{|c|}{ Single-gland weight ${ }^{\mathrm{a}, \mathrm{b}}(\mathrm{g})(n=222)$} & $0.481(0.007-13.5)$ \\
\hline \multicolumn{3}{|c|}{ Note:-PTH indicates parathyroid hormone. } \\
\hline & $\begin{array}{l}\text { Single-Gland } \\
\text { Localization }\end{array}$ & $\begin{array}{l}\text { Single-Gland } \\
\text { Lateralization }\end{array}$ \\
\hline Sensitivity, \% & $55.4 \pm 3.5$ & $78.8 \pm 2.8$ \\
\hline Specificity, \% & $85.9 \pm 1.4$ & $67.8 \pm 3.2$ \\
\hline Positive predictive value, $\%$ & $56.8 \pm 3.4$ & $71.0 \pm 2.7$ \\
\hline Negative predictive value, $\%$ & $85.3 \pm 1.1$ & $76.2 \pm 3.1$ \\
\hline
\end{tabular}

assessed when information was available: body mass index of $\geq 30$, history of diabetes, prior neck surgery other than parathyroid surgery, presence of hypothyroidism, presence of thyroid goiter or nodules, history of prior radioiodine treatment for thyroid disease, gland weight $(>1 \mathrm{~g})$, preoperative parathyroid hormone level ( $\geq 150)$, and calcium level ( $\geq 11)$. A test from sampling theory was used for the independent estimates of sensitivity in the 2 groups. This test was performed separately for localization and lateralization.

\section{RESULTS}

\section{Patients}

Between 2009 and 2013, 278 patients met the inclusion criteria. Table 1 shows the descriptive data regarding our patient population.

\section{Localization and Lateralization}

Of the 278 patients, $48(17.3 \%)$ had multigland disease found at surgery and 8 had single glands located outside the 4 quadrants. These patients were excluded from localization and lateralization analysis, leaving 222 patients with single-gland disease in 1 of the 4 quadrants.

The sensitivity and specificity of 2-phase parathyroid CT to correctly localize a single gland to 1 of the 4 quadrants were $55.4 \% \pm 3.5 \%$ and $85.9 \% \pm 1.4 \%$, respectively (Table 2 ). The positive and negative predictive values for single-gland localization were $56.8 \% \pm 3.4 \%$ and $85.3 \% \pm 1.1 \%$, respectively.

The sensitivity and specificity of 2-phase parathyroid CT to correctly lateralize a single gland were $78.8 \% \pm 2.8 \%$ and $67.8 \% \pm 3.2 \%$, respectively. The positive and negative predictive values for single-gland localization were $71.0 \% \pm 2.7 \%$ and $76.2 \% \pm 3.1 \%$, respectively.

We further evaluated the sensitivities and specificities for localization and lateralization for single adenomas on the basis of specific quadrants. These data are provided in Table 3.

AJNR Am J Neuroradiol 36:2373-79 Dec 2015 www.ajnr.org 
Multigland Disease and Nontraditional Location Glands

The ability of 2-phase parathyroid CT to correctly identify the presence of multiple glands was also assessed. For the 48 patients with multiglandular disease, the sensitivity and specificity of the 2-phase parathyroid CT to correctly identify their disease was $22.9 \% \pm 6.1 \%$ and $79.5 \% \pm 2.7 \%$, respectively. The positive and negative predictive values were $19.0 \% \pm 5.1 \%$ and $83.1 \% \pm 2.5 \%$.

Eight patients were identified as having nontraditionally located glands at the time of surgery, including 4 patients with glands identified within the superior mediastinum, 1 patient with an inferiorly descended superior parathyroid gland in a right paratracheal location, 1 patient with a gland located within the left piriform sinus, and 2 patients with intrathyroidal parathyroid adenomas. Mediastinal or ectopic glands in all 6 patients were correctly identified, though the piriform sinus lesion was not prospectively inferred to be an adenoma. The 2 intrathyroidal adenomas were not identified.

\section{Clinical Factors Affecting Accuracy}

A variety of clinical factors in patients with single adenomas was evaluated for their effects on sensitivity and specificity for local- ization and lateralization (Table 4). For localization, only the gland weight of $\geq 1 \mathrm{~g}$ led to a higher sensitivity $(69.8 \%$ versus $50.0 \%, P=.014)$. The group with a calcium level $\geq 11$ had a higher sensitivity, $62.7 \%-49.7 \%$, which demonstrated a trend toward statistical significance $(P=.070)$. There were no other statistically significant results.

Similarly, patients with a gland weight of $\geq 1$ g or a body mass index of $<30$ demonstrated higher sensitivity for lateralization (90.7\% versus $71.9 \%, P=.001$, and $84.0 \%$ versus $69.2 \%, P=$ .022 , respectively). In addition, patients with a history of hypothyroidism had a lower sensitivity than those without (59.4\% versus $77.8 \%, P=.049)$ and lower specificity ( $46.9 \%$ versus $66.1 \%$, $P=.046)$.

\section{DISCUSSION}

Between $0.2 \%$ and $0.5 \%$ of the population are affected by primary hyperparathyroidism, with approximately 100,000 new cases diagnosed annually in the United States. ${ }^{17}$ In the past, treatment of primary hyperparathyroidism required subjecting a patient to bilateral cervical exploration. In recent years, however, treatment has shifted toward the use of minimally invasive, or focused, parathyroidectomy. A 2011 study by Udelsman et al $^{3}$ evaluating 1650 patients undergoing surgery for primary hyperparathyroidism found that pa-

Table 3: Quadrant-specific single-gland detection accuracy

\begin{tabular}{lcccc} 
& Right Upper & Right Lower & Left Upper & Left Lower \\
\hline Sensitivity, \% & $26.2 \pm 6.9$ & $73.6 \pm 6.1$ & $51.0 \pm 7.1$ & $63.8 \pm 6.4$ \\
Specificity, \% & $70.6 \pm 3.4$ & $95.6 \pm 2.0$ & $83.0 \pm 2.9$ & $90.8 \pm 2.3$ \\
Positive predictive value, \% & $79.2 \pm 2.2$ & $89.8 \pm 6.1$ & $50.0 \pm 6.4$ & $69.8 \pm 6.2$ \\
Negative predictive value, \% & $76.2 \pm 3.1$ & $91.6 \pm 1.8$ & $83.6 \pm 2.2$ & $88.3 \pm 1.9$ \\
\hline
\end{tabular}
parathyroidectomy demonstrated improved cure and complication rates and a decreased length of hospital stay and lower total hospital charges.

Table 4: Effect of clinical variables on sensitivity and specificity of localization and lateralization in patients with single adenomas

\begin{tabular}{|c|c|c|c|c|c|c|}
\hline & \multicolumn{3}{|c|}{ Localization } & \multicolumn{3}{|c|}{ Lateralization } \\
\hline & Variable: Yes & Variable: No & $P$ Value & Variable: Yes & Variable: No & $P$ Value \\
\hline \multicolumn{7}{|c|}{$\mathrm{BMI} \geq 30\left(n^{\mathrm{a}}=166\right)$} \\
\hline Sensitivity, \% & 48.4 & 56.0 & .328 & 69.2 & 84.0 & .022 \\
\hline Specificity, \% & 87.9 & 82.2 & .081 & 59.3 & 69.3 & .180 \\
\hline \multicolumn{7}{|l|}{ Diabetes $(n=213)$} \\
\hline Sensitivity, \% & 45.2 & 53.3 & .407 & 71.0 & 75.3 & .628 \\
\hline Specificity, \% & 88.2 & 85.7 & .456 & 61.3 & 63.7 & .799 \\
\hline \multicolumn{7}{|c|}{ Prior neck surgery $(n=201)$} \\
\hline Sensitivity, \% & 55.0 & 53.0 & .870 & 75.0 & 75.7 & .947 \\
\hline Specificity, \% & 91.7 & 85.6 & .99 & 75.0 & 63.5 & .278 \\
\hline \multicolumn{7}{|c|}{ Hypothyroidism $(n=212)$} \\
\hline Sensitivity, \% & 43.8 & 54.4 & .268 & 59.4 & 77.8 & .049 \\
\hline Specificity, \% & 86.5 & 85.7 & .872 & 46.9 & 66.1 & .046 \\
\hline \multicolumn{7}{|c|}{ Thyroid goiter or nodules $(n=218)$} \\
\hline Sensitivity, \% & 46.4 & 57.0 & .143 & 69.6 & 78.5 & .170 \\
\hline Specificity, \% & 87.4 & 85.7 & .540 & 58.0 & 67.1 & .199 \\
\hline \multicolumn{7}{|c|}{ Radioactive iodine therapy $(n=218)$} \\
\hline Sensitivity, \% & 42.9 & 53.6 & .602 & 57.1 & 75.8 & .360 \\
\hline Specificity, \% & 81.0 & 86.3 & .596 & 42.9 & 64.5 & .291 \\
\hline \multicolumn{7}{|c|}{ Gland weight $\geq \lg (n=221)$} \\
\hline Sensitivity, \% & 69.8 & 50.0 & .014 & 90.7 & 71.9 & .001 \\
\hline Specificity, \% & 85.3 & 86.5 & .736 & 72.1 & 62.4 & .213 \\
\hline \multicolumn{7}{|c|}{ Preoperative PTH level $\geq 150(n=221)$} \\
\hline Sensitivity, \% & 53.8 & 53.1 & .911 & 81.3 & 71.5 & .087 \\
\hline Specificity, \% & 84.6 & 87.7 & 0.274 & 64.8 & 64.6 & 0.973 \\
\hline \multicolumn{7}{|c|}{ Preoperative calcium level $\geq 11(n=222)$} \\
\hline Sensitivity, \% & 62.7 & 49.7 & 0.070 & 80.6 & 73.5 & 0.242 \\
\hline Specificity, \% & 86.1 & 86.2 & 0.957 & 67.2 & 63.2 & 0.572 \\
\hline
\end{tabular}

Note:-BMI indicates body mass index; PTH, parathyroid hormone.

${ }^{a}$ Total number of patients in whom this variable could be confirmed on retrospective chart review. 


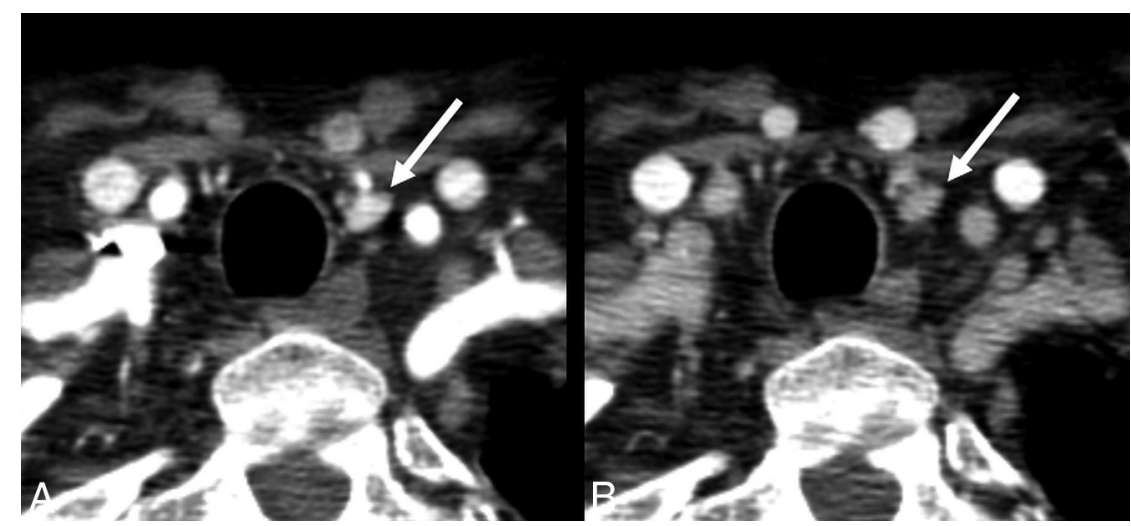

FIG 2. Axial arterial phase $(A)$ and axial venous phase $(B)$ images show a left inferior parathyroid adenoma, which demonstrates avid early contrast enhancement on the arterial phase and washout on the venous phase.

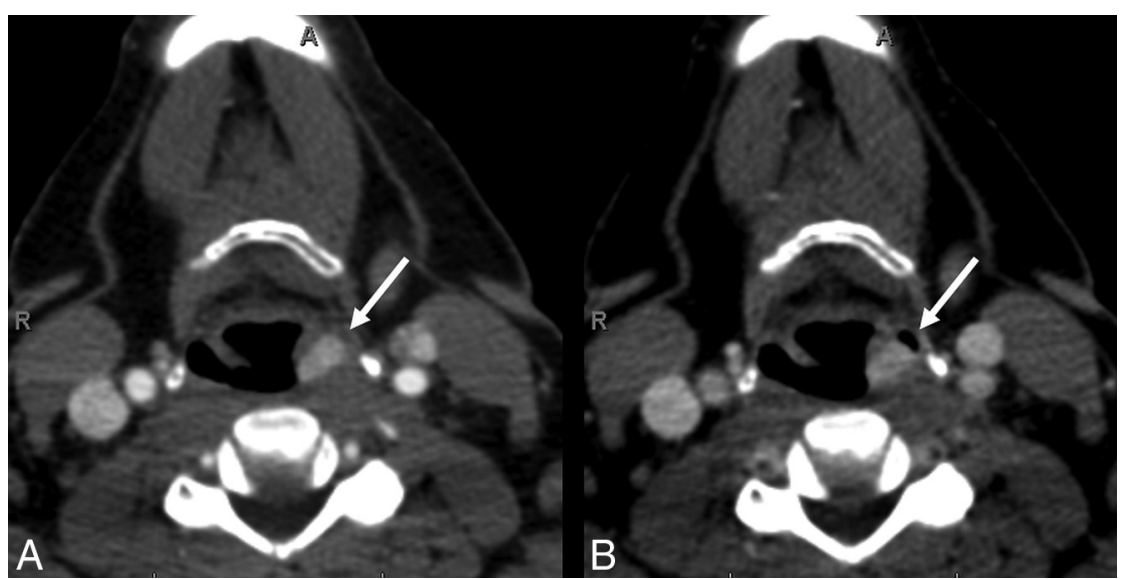

FIG 3. Axial arterial phase $(A)$ and axial venous phase $(B)$ images show an ectopic superior left parathyroid adenoma, which was surgically found to be within the submucosa of the left piriform sinus.

The success of minimally invasive parathyroidectomy depends on the appropriate selection of candidates-namely, those with localizable adenomas on preoperative imaging. ${ }^{18}$ A number of imaging modalities, including radionuclide scintigraphy, sonography, and $\mathrm{CT}$, are currently used, both alone and in combination, for localization purposes, with varying levels of success.

A prior meta-analysis of 20,225 cases of primary hyperparathyroidism demonstrated radionuclide scintigraphy having a sensitivity of $88 \%$ for detecting solitary adenomas, ${ }^{19}$ though the sensitivity decreased to between $51 \%$ and $69 \%$ for adenomas of $\leq 500$ mg. ${ }^{20,21}$ Similarly, sonography is reported to have sensitivities for lateralization ranging from $61 \%$ to $88 \%$. Many consider sonography to be the first-line imaging study, due to its ability to localize enlarged parathyroid glands while concurrently determining the presence of relevant thyroid disease. Both of these modalities are plagued by poor spatial resolution, however, and sonography is limited in its ability to adequately visualize the mediastinum. ${ }^{13}$ In addition, sonography is user-dependent and, as such, is limited by the skill and experience of the ultrasonographer. ${ }^{22}$

Regardless of the preferred technique, in select patients, neither sonography nor radionuclide scintigraphy provides adequate localizing information. These patients may benefit from further attempts at preoperative localization. In addition, because of in- dividual patient characteristics, such as high body mass index, a surgeon may want additional imaging studies.

In recent years, multiphase CT has been increasingly used at our institution as a means of localizing adenomas before surgery in select patients. The ability of multiphase CT to identify parathyroid adenomas is dependent on differences in enhancement characteristics between parathyroid lesions and other soft-tissue structures in the neck, with parathyroid lesions demonstrating rapid uptake and washout of contrast (Figs 2 and 3) versus the progressive enhancement pattern seen in normal lymph nodes. ${ }^{12}$ In addition, CT has the added benefit of providing the surgeon with exquisite anatomic detail that can be used for surgical planning.

In 2006, 4D-CT was first reported in the literature as a means of identifying parathyroid adenomas. In that study, patients underwent sestamibi imaging, sonography, and $4 \mathrm{D}-\mathrm{CT}$, which consisted of precontrast, postcontrast, and delayed images. ${ }^{9}$ Investigators demonstrated the improved sensitivity $(88 \%)$ of $4 \mathrm{D}$-CT compared with sestamibi imaging (65\%) and sonography (57\%) for lateralizing hyperfunctioning parathyroid glands to 1 side of the neck. ${ }^{9}$ In addition, the sensitivity of 4D-CT in localizing parathyroid tumors to the correct quadrant of the neck was $70 \%$ compared with only $33 \%$ and $29 \%$ for sestamibi and sonography, respectively. ${ }^{9}$

Since that initial report by Rodgers et al in $2006,{ }^{9}$ many studies have attempted to determine the number of imaging phases needed to optimize parathyroid adenoma detection. A 2011 study by Starker et $\mathrm{al}^{10}$ found that a $4 \mathrm{D}-\mathrm{CT}$ consisting of 4 phases (precontrast followed by imaging at 30,60, and 120 seconds following intravenous contrast injection) had improved sensitivity (85.7\%) compared with sestamibi with SPECT (40.4\%) and ultrasound (48.0\%) for localizing parathyroid adenomas to the correct quadrant of the neck. That study showed similar superiority of $4 \mathrm{D}-\mathrm{CT}$ for lateralizing parathyroid lesions as well $(93.9 \%$ for $4 \mathrm{D}-\mathrm{CT}$, $71.2 \%$ for ultrasound, $61.5 \%$ for sestamibi with SPECT). ${ }^{10}$ Similarly, a study by Eichhorn-Wharry et $\mathrm{al}^{14}$ found 4 -phase CT to be significantly more sensitive than sestamibi in correctly lateralizing parathyroid adenomas ( $73 \%$ versus $62 \%)$, with an even greater difference seen in patients with serum calcium levels of $<10.8$ $\mathrm{mg} / \mathrm{dL}$ ( $45 \%$ versus $29 \%$ ). Additional studies by Beland et $\mathrm{al}^{12}$ and Hunter et $\mathrm{al}^{13}$ also evaluated $4 \mathrm{D}$-CT with 4 imaging phases. In the former, investigators found a sensitivity of $82 \%$ and a specificity of $92 \%$. In the latter, Hunter et al found 4D-CT to have a $93.7 \%$ accuracy for correct lateralization and an $86.6 \%$ accuracy for quadrant localization.

AJNR Am J Neuroradiol 36:2373-79 Dec 2015 www.ajnr.org 
Investigators have also evaluated the use of 3-phase 4D-CT. Chazen et al, ${ }^{23}$ by using 3 phases consisting of precontrast, postcontrast, and delayed, found a sensitivity for correct lateralization of $93 \%$ and correct localization of $92 \%$ in 32 pathologically proved cases of single parathyroid adenomas. Similarly, Sepahdari et $\mathrm{al}^{24}$ reported $97 \%$ accuracy for single-gland disease in a 3 -phase protocol with precontrast, arterial, and venous phase imaging.

Finally, Kutler et $\mathrm{al}^{11}$ used a modified technique of 4D-CT/ sonography to identify parathyroid adenomas and found that it had a sensitivity and specificity of $94 \%$ and $96 \%$, respectively, for lateralizing hyperfunctioning parathyroid glands and $82 \%$ and $93 \%$, respectively, for localizing abnormal parathyroid glands to 1 quadrant of the neck. A similar study found that this technique demonstrated a good sensitivity for localization even in adenomas weighing $<150 \mathrm{mg}^{1}$

Despite high reported localization success rates, 4D-CT remains burdened by the substantial radiation dose associated with scanning the patient multiple times. In addition, the optimal number of phases and the timing of contrast are still under investigation. In fact, a recent study by Raghavan et $\mathrm{al}^{16}$ evaluated the accuracy of different combinations of CT phases to accurately localize parathyroid adenomas in 29 patients with primary hyperparathyroidism and found that the lateralization and localization accuracy of the arterial phase alone were comparable with that in other combinations of phases.

Our study, which includes 278 patients with surgically cured primary hyperparathyroidism, is the largest to date evaluating the accuracy of multiphase CT in preoperatively localizing pathologic parathyroid glands. Compared with the literature, our findings demonstrate a more modest success rate of our 2-phase CT protocol, with an overall sensitivity of $55.4 \%$ and specificity of $85.9 \%$ for localizing disease to a specific quadrant, which are less than those reported by others in the literature. $^{1,9,10,12,13,23}$ Similarly, the success of 2-phase CT to correctly lateralize adenomas, with a sensitivity and specificity of $78.8 \%$ and $67.8 \%$, respectively, is also less than that reported for 4-phase CT in the literature.

A number of factors could account for the decreased accuracy of 2-phase CT in this study. The most important is related to study design, because this was a retrospective analysis designed specifically to reflect the performance of 2-phase-CT in a true clinical setting. As such, the study is prone to the same limitations that affect radiologists' interpretations on a routine clinical basis. These include issues related to the following: 1) patient factors, such as streak artifacts from the shoulders and clavicles, and artifacts related to breathing and swallowing; 2) gland-specific factors that may hinder accurate characterization by dynamic CT, such as in the case of cystic adenomas; 3 ) technical factors related to the CT scan itself, such as poor contrast bolus timing and streak artifacts due to venous contrast pooling; and 4) finally, interpretation-related factors, such as differences in reader experience and skill in interpreting 2-phase studies. Although these factors influence the sensitivity and specificity of parathyroid CT studies regardless of the number of phases performed, these may be less apparent in more controlled settings with smaller sample sizes of patients and smaller numbers of more experienced readers.

An additional factor that could potentially affect the accuracy of this 2-phase protocol in identifying parathyroid adenomas is problems with differentiating juxtathyroidal adenomas from thyroid tissue because both can have similar contrast-enhancement characteristics. As such, the lack of a precontrast phase may limit the ability to accurately identify juxtathyroidal adenomas, particularly in the setting of multinodular goiter, but our analysis of clinical factors affecting the accuracy of detection did not find a difference between patients with multinodular goiter and those without.

Additional factors in this study must also be taken into account as well. First, the 4-quadrant classification system used in this study is unable to differentiate an inferior parathyroid adenoma from a superior parathyroid adenoma that has overly descended along the tracheoesophageal groove. At imaging, this gland would be classified in the inferior quadrant due to its relationship with the thyroid, while at surgery it would be classified as a superior gland. This study did not attempt to differentiate these glands on imaging. This limitation is reflected in the quadrantspecific analysis of sensitivity, with the upper quadrants demonstrating lower sensitivities compared with the lower quadrants (26.2\% and $51.0 \%$ versus $73.6 \%$ and $63.8 \%)$. An additional limitation relates to the study-selection criteria-in particular, exclusion of 18 patients who were not cured following surgery. Unfortunately, this limitation is due to the retrospective design of the study and was necessary because surgical localization was treated as the criterion standard, without which the accuracy of the technique could not be determined. However, this failed cure rate of $6.1 \%$ (18 of 296 patients) is not much different from the failure rate of up to $5 \%$ often reported in the literature, and the small difference may reflect a slightly more surgically challenging patient population requiring 2-phase CT. ${ }^{25-27}$ Similarly, patients undergoing repeat surgery ( 12 of 296 patients) were excluded because the purpose of this study was to evaluate the accuracy of 2-phase CT in patients at initial presentation.

\section{CONCLUSIONS}

Two-phase parathyroid CT offers an additional potential method for the preoperative localization of parathyroid adenomas with sensitivities for lateralization and quadrant-specific localization of $78.8 \%$ and $55.4 \%$, respectively. Despite lower accuracy rates than those of $4 \mathrm{D}-\mathrm{CT}$ techniques in the literature, the lower accuracy rates must be balanced with the potential reduction in radiation dose. In addition, the clinical environment in which this study was performed, which subjects radiologists to the same limitations routinely encountered during study interpretation, may more fairly represent the accuracy of this technique when implemented in everyday clinical practice. Further large cohort prospective studies are needed to definitively determine the optimal number of phases required and the appropriate population for each technique.

\section{REFERENCES}

1. Stucken EZ, Kutler DI, Moquete R, et al. Localization of small parathyroid adenomas using modified 4-dimensional computed to- 
mography/ultrasound. Otolaryngol Head Neck Surg 2012;146:33-39 CrossRef Medline

2. Ellika S, Patel S, Aho T, et al. Preoperative localization of parathyroid adenomas using 4-dimensional computed tomography: a pictorial essay. Can Assoc Radiol J 2013;64:258 -68 CrossRef Medline

3. Udelsman R, Lin Z, Donovan P. The superiority of minimally invasive parathyroidectomy based on 1650 consecutive patients with primary hyperparathyroidism. Ann Surg 2011;253:585-91 CrossRef Medline

4. Udelsman R. Six hundred fifty-six consecutive explorations for primary hyperparathyroidism. Ann Surg 2002;235:665-70; discussion 670-72 CrossRef Medline

5. Vitetta GM, Neri P, Chiecchio A, et al. Role of ultrasonography in the management of patients with primary hyperparathyroidism: retrospective comparison with technetium-99m sestamibi scintigraphy. J Ultrasound 2014;17:1-12 CrossRef Medline

6. Grosso I, Sargiotto A, D'Amelio P, et al. Preoperative localization of parathyroid adenoma with sonography and $99 \mathrm{mTc}$-sestamibi scintigraphy in primary hyperparathyroidism. J Clin Ultrasound 2007; 35:186-90 CrossRef Medline

7. Reeder SB, Desser TS, Weigel RJ, et al. Sonography in primary hyperparathyroidism: review with emphasis on scanning technique. J Ultrasound Med 2002;21:539-52; quiz 553-54 Medline

8. Hoang JK, Sung WK, Bahl M, et al. How to perform parathyroid 4D CT: tips and traps for technique and interpretation. Radiology 2014; 270:15-24 CrossRef Medline

9. Rodgers SE, Hunter GJ, Hamberg LM, et al. Improved preoperative planning for directed parathyroidectomy with 4-dimensional computed tomography. Surgery 2006;140:932-40; discussion 940-41 CrossRef Medline

10. Starker LF, Mahajan A, Björklund $\mathrm{P}$, et al. 4D parathyroid CT as the initial localization study for patients with de novo primary hyperparathyroidism. Ann Surg Oncol 2011;18:1723-28 CrossRef Medline

11. Kutler DI, Moquete R, Kazam E, et al. Parathyroid localization with modified 4D-computed tomography and ultrasonography for patients with primary hyperparathyroidism. Laryngoscope 2011;121: 1219-24 CrossRef Medline

12. Beland MD, Mayo-Smith WW, Grand DJ, et al. Dynamic MDCT for localization of occult parathyroid adenomas in 26 patients with primary hyperparathyroidism. AJR Am J Roentgenol 2011;196:61-65 CrossRef Medline

13. Hunter GJ, Schellingerhout D, Vu TH, et al. Accuracy of four-dimensional CT for the localization of abnormal parathyroid glands in patients with primary hyperparathyroidism. Radiology 2012;264: 789-95 CrossRef Medline

14. Eichhorn-Wharry LI, Carlin AM, Talpos GB. Mild hypercalcemia: an indication to select 4-dimensional computed tomography scan for preoperative localization of parathyroid adenomas. Am J Surg 2011; 201:334-38; discussion 338 CrossRef Medline
15. US Food and Drug Administration. Initiative to Reduce Unnecessary Radiation Exposure from Medical Imaging. Updated March 24, 2015. http://www.fda.gov/Radiation-emittingProducts/RadiationSafety/RadiationDoseReduction/default.htm. Accessed March 24, 2015

16. Raghavan P, Durst CR, Ornan DA, et al. Dynamic CT for parathyroid disease: are multiple phases necessary? AJNR Am J Neuroradiol 2014;35:1959-64 CrossRef Medline

17. Pruhs ZM, Starling JR, Mack E, et al. Changing trends for surgery in elderly patients with hyperparathyroidism at a single institution. J Surg Res 2005;127:58-62 CrossRef Medline

18. van Dalen A, Smit CP, van Vroonhoven TJ, et al. Minimally invasive surgery for solitary parathyroid adenomas in patients with primary hyperparathyroidism: role of US with supplemental CT. Radiology 2001;220:631-39 CrossRef Medline

19. Ruda JM, Hollenbeak CS, Stack BC Jr. A systematic review of the diagnosis and treatment of primary hyperparathyroidism from 1995 to 2003. Otolaryngol Head Neck Surg 2005;132:359-72 CrossRef Medline

20. Bergenfelz A, Tennvall J, Valdermarsson S, et al. Sestamibi versus thallium subtraction scintigraphy in parathyroid localization: a prospective comparative study in patients with predominantly mild primary hyperparathyroidism. Surgery 1997;121:601-05 CrossRef Medline

21. Jones JM, Russell CF, Ferguson WR, et al. Pre-operative sestamibi-technetium subtraction scintigraphy in primary hyperparathyroidism: experience with 156 consecutive patients. Clin Radiol 2001;56:556-59 CrossRef Medline

22. Steward DL, Danielson GP, Afman CE, et al. Parathyroid adenoma localization: surgeon-performed ultrasound versus sestamibi. $L a$ ryngoscope 2006;116:1380-84 CrossRef Medline

23. Chazen JL, Gupta A, Dunning A, et al. Diagnostic accuracy of 4D-CT for parathyroid adenomas and hyperplasia. AJNR Am J Neuroradiol 2012;33:429-33 CrossRef Medline

24. Sepahdari AR, Yeh MW, Rodrigues D, et al. Three-phase parathyroid 4-dimensional computed tomography initial experience: inexperienced readers have high accuracy and high interobserver agreement. J Comput Assist Tomogr 2013;37:511-17 CrossRef Medline

25. Boggs JE, Irvin III GL, Carneiro DM, et al. The evolution of parathyroidectomy failures. Surgery 1999;126:998-1002; discussion 1002-3 Medline

26. Carneiro DM, Solorzano CC, Irvin III GL. Recurrent disease after limited parathyroidectomy for sporadic primary hyperparathyroidism. J Am Coll Surg 2004;199:849-53; discussion 853-55 CrossRef Medline

27. Lew JI, Rivera M, Irvin III GL, et al. Operative failure in the era of focused parathyroidectomy. Arch Surg 2010;145:628-33 CrossRef Medline 